\title{
RELAÇÃO DO PADRÃO SAZONAL DA VEGETAÇÃO COM A PRECIPITAÇÃO NA REGIÃO DE CERRADO DA AMAZÔNIA LEGAL, USANDO ÍNDICES ESPECTRAIS DE VEGETAÇÃO
}

\author{
JORGE ALBERTO BUSTAMANTE BECERRA ${ }^{1}$, YOSIO EDEMAR SHIMABUKURO² E \\ REGINA CÉLIA DOS SANTOS ALVALÁ ${ }^{1}$
}

\author{
Instituto Nacional de Pesquisas Espaciais (INPE) \\ ${ }^{1}$ Centro de Ciência do Sistema Terrestre (CCST), Cachoeira Paulista, SP, Brasil \\ ${ }^{2}$ Grupo de Observação da Terra (OBT), São José dos Campos, SP, Brasil
}

jorge.bustamante@cptec.inpe.br, yosio@dsr.inpe.br, regina@cptec.inpe.br

Recebido Março 2008 - Aceito Dezembro 2008

\begin{abstract}
RESUMO
A precipitação é um dos principais fatores que determina a dinâmica sazonal da vegetação na região de savanas tropicais, como é o caso do cerrado brasileiro. Neste trabalho foram analisadas as relações da precipitação sazonal, com o comportamento sazonal das classes de uso e cobertura da terra (UCT), principalmente as fisionomias de cerrado do Estado de Tocantins. Foi analisada a dinâmica sazonal do cerrado, incluindo áreas florestadas e não florestadas, a partir da análise de imagens do MODIS/TERRA IV (Índices de Vegetação) de janeiro a dezembro de 2004, bem como dados diários de precipitação de 2004 e uma série de precipitação diária do período de 1969 a 2005. Os resultados da análise de precipitação mostram que a área de estudo apresentou uma alta sazonalidade, com estação seca de maio a setembro. As análises dos IV mostram que a dinâmica sazonal das formações de cerrado é similar àquela das áreas convertidas para outros usos. O padrão sazonal das classes de UCT segue os padrões da precipitação, cujos menores valores foram registrados no mês de agosto de 2004, mês este que apresentou os menores valores dos IV. Diferentemente das demais classes de UCT, a formação florestal não se ajustou ao padrão de precipitação, apresentando valores de IV similares ao longo do ano com leve decréscimo no mês de setembro de 2004.
\end{abstract}

Palavra chave: Uso e cobertura da terra, índices espectrais de vegetação, precipitação, vegetação sazonal do cerrado.

ABSTRACT: RELATIONSHIP BETWEEN VEGETATION SEASONAL PATTERN AND
PRECIPITATION IN THE CERRADO REGION BY SPECTRAL VEGETATION INDEXES
Precipitation is one of the main factors that determine the seasonal dynamics of the vegetation in tropical
savanna areas, as the Brazilian cerrado. In this work the relationship of the seasonal precipitation with
the seasonal behavior of the land use and land cover (LULC) types, mainly savannah physiognomies
of the Tocantins State, was investigated. We analyzed the savanna seasonal dynamics, including forest
and converted areas, with MODIS/TERRA VI (vegetation indexes) satellite measurements from
January to December 2004 and daily precipitation of 2004 and daily precipitation series from 1969 to
2005 . The results of the precipitation analysis show that the study areas exhibited a high seasonality
with a dry season from May to September. The analysis of the VI showed that the seasonal dynamic
of the savanna formations and the converted areas were similar. The seasonal pattern of these LULC
types follows the precipitation pattern, when the lowest VI values were recorded in August of 2004.
Differently of these LULC types, forest formations do not fitted to the precipitation pattern, this LULC
type keep similar VI values along year with light decrease in September of 2004 . Keywords: Land use and land cover, vegetation indexes, precipitation, seasonal vegetation of tropical savanna. 


\section{INTRODUÇÃO}

O desenvolvimento de modelos de circulação geral (GCMs) e de esquemas de transferência solo-plantaatmosfera (SVATs) acoplados aos GCMs, têm contribuído significativamente para a melhoria das previsões de tempo e de clima (Sellers et al., 1986; Xue e Shukla, 1993). Variações nas características das superfícies continentais podem ter impactos significativos no clima, isto é, a atmosfera é sensível ao albedo da superfície, à umidade do solo, à rugosidade, e outras características da superfície em muitas escalas de tempo (Charney et al., 1977; Shukla e Mintz, 1982; Sud et al., 1988). As propriedades morfológicas da vegetação, tais como o índice de área foliar, ângulo de distribuição das folhas e coeficientes de espalhamento das folhas e do solo, são importantes para determinar as características da superfície continental e sua variação; portanto, afetam as interações entre a biosfera terrestre e a atmosfera, especificamente os fluxos de radiação, de momentum, de calor sensível e de calor latente.

Embora os SVATs apresentem uma descrição detalhada das interações entre a superfície continental e a atmosfera, a representação inadequada ou incompleta dos processos de superfície pode ter um impacto negativo sobre a previsão de tempo e estudos climáticos. Assim, esforços têm sido despendidos no intuito de se buscar uma representação mais realista da vegetação (Wilson e Henderson-Sellers, 1985; Hall et al., 1995; Sud et al., 1996; DeFries et al., 1999; Champeaux et al., 2000), bem como no aprimoramento do conhecimento dos detalhes regionais dos diversos processos na atmosfera e suas interações com o ciclo hidrológico.

No Centro de Previsão de Tempo e Estudos Climáticos do Instituto Nacional de Pesquisas Espaciais (CPTEC/INPE), informações sobre a vegetação para a modelagem climática da Amazônia, provêm do mapa de vegetação elaborado no âmbito do projeto ProVeg (Sestini et al., 2002). Apesar do esforço empreendido, o mapa de cobertura e uso da terra da região da Amazônia legal gerado por Sestini et al. (2002), não leva em consideração a antropização de áreas de cerrado, que nas últimas décadas têm aumentado tanto em extensão (pastagem para pecuária) quanto em intensidade (culturas de ciclo curto).

Outra característica essencial que não é levada em conta é a marcada sazonalidade desta região (cerrado), com perda total ou parcial de folhas na estação seca (Ratter et al., 1997) e produção de novas folhas e aceleração dos processos metabólicos na estação chuvosa. Essa alternância é comandada pela disponibilidade hídrica.

O Bioma Cerrado (savana tropical brasileira) representa o segundo maior bioma brasileiro, cobrindo $23 \%$ (dois milhões de quilômetros quadrados) do território nacional. Este Bioma é um complexo de formações vegetais, que apresenta fisionomias e composição florística variáveis: campestres (campo limpo), savânicas (campo sujo, campo cerrado e cerrado stricto sensu$s s$ ), e florestais (cerradão), formando um mosaico ecológico, (Coutinho, 1978). Além destas fitofisionomias, florestas de galeria e florestas decíduas compõem este bioma.

Nos últimos 35 anos tem sido desenvolvida uma agricultura moderna para produção de soja, milho e arroz, entre outros cultivos, bem como a criação de grandes rebanhos de gado em pastagens naturais e cultivadas na região do Bioma Cerrado. Além destas classes de ocupação em detrimento da cobertura natural da região, existem desflorestamentos para produção de carvão e muitos incêndios provocados para posterior uso das áreas queimadas.

Os efeitos da diminuição da cobertura arbórea das savanas do globo no clima regional foram analisados por Hoffmann e Jackson (2000). Com base em simulações realizadas com o modelo de circulação geral CCM3 acoplado ao modelo de esquema de superfície NCAR, determinaram que a substituição da cobertura arbórea por pastagens reduz em aproximadamente $10 \%$ a precipitação, com incrementos da freqüência de períodos secos na estação chuvosa, além de um incremento médio da temperatura do ar à superfície de $0,5^{\circ} \mathrm{C}$.

A importância do clima, tanto na estrutura como na função da vegetação é amplamente conhecida (Walter, 1973; Box, 1981). A distribuição espacial e estrutura vertical da vegetação natural são determinadas pela interação de fatores ambientais abióticos e bióticos, como clima, solo, geomorfologia e fauna associada a estes ambientes naturais. Estas interações permitem também que a cobertura vegetal tenha um papel importante nos sistemas climáticos, devido a trocas de energia, água e gases com a atmosfera e também como fonte de produção e seqüestro de gases no ciclo biogeoquímico (Sellers et al., 1997). Segundo Shukla et al. (1990), o equilíbrio dinâmico existente entre vegetação e clima regional pode ser alterado se um dos seus componentes mudar. Na América do Sul, mais especificamente no Bioma Cerrado parecem ser aparentes perturbações em ambos componentes (vegetação e clima).

As formações vegetais, que compõem o Bioma Cerrado, encontram-se extremamente fragilizadas devido à substituição acelerada do Cerrado pela agropecuária, principalmente na região Centro-Oeste do país incluindo o Estado de Tocantins.

Levando em consideração que a pressão antrópica no Bioma Cerrado vem aumentando, o que significa substituição de formações campestres, savânicas e até florestais, por áreas destinadas à agropecuária, é importante determinar a composição fitofisionômica do Cerrado para posterior avaliação de como as mudanças do uso da terra e do clima afetam as funções biológicas, químicas e físicas dos ecossistemas amazônicos. Ressalta-se que o Bioma Cerrado no Estado de Tocantins, encontra-se adjacente à Floresta Amazônica e faz 
parte da Amazônia Legal, compondo assim parte do grande ecossistema amazônico.

No que concerne aos mapeamentos do uso e cobertura da terra do Bioma Cerrado da Amazônia Legal para modelagem meteorológica, em alguns casos usaram-se imagens do Landsat (Sestini et al., 2002) ou imagens do MODIS, como o DETER (2005); no entanto, estes mapeamentos agrupam as diferentes fisionomias do Cerrado em uma única classe, devido à dificuldade na sua identificação pela confusão com outras classes de cobertura vegetal.

Um dos principais fatores climáticos que influencia a ocorrência e distribuição espacial e temporal das fisionomias de cerrado é a precipitação pluvial. Trabalhos recentes mostram a importância da análise temporal ou sazonal da vegetação de cerrado (Ferreira et al., 2003; Ferreira e Huete, 2004; Ratana e Huete, 2004; Becerra e Alvalá, 2006) a partir de séries temporais de índices espectrais de vegetação. Mais recentemente, Huete et al., (2006), analisaram a relação direta do padrão sazonal da precipitação com a sazonalidade da vegetação.

Neste contexto, o objetivo do presente trabalho é classificar a cobertura vegetal do Estado de Tocantins, que compõe uma parte do Bioma Cerrado na Amazônia Legal, e determinar a relação do padrão sazonal de precipitação (ano de 2004) com a dinâmica sazonal da vegetação, a partir da aplicação de dois índices espectrais de vegetação, NDVI e EVI, e a análise de seis classes de UCT (floresta, cerradão, cerrado ss, campo cerrado, agricultura e agricultura-pastagem). A premissa a ser testada é que as diferentes classes de uso e cobertura da terra, especialmente as fisionomias de cerrado, respondem de forma diferenciada aos padrões sazonais da precipitação. Isto significa que fisionomias vegetais com maior disponibilidade hídrica, devem sofrer menos em períodos de menor precipitação (estação seca), e fisionomias com menor disponibilidade hídrica, devem sofrer mais nesta mesma época do ano.

\section{MATERIAIS E MÉTODOS}

\section{1 Área de estudo}

A área de estudo corresponde ao Estado de Tocantins (Figura 1), localizada no sudeste da Amazônia Legal Brasileira, entre as coordenadas $05^{\circ} 09^{\prime} 59,25^{\prime \prime}$ e $13^{\circ} 27^{\prime} 55,54^{\prime \prime}$ de latitude Sul e 45०41'41,93" e 5044'38,36" de longitude Oeste. O clima da região é tropical com estação seca de maio a outubro. $\mathrm{Na}$ região norte do Estado a precipitação é intensa, diminuindo para o sul e sudeste (Nimer, 1977).

Em Tocantins predomina o Bioma Cerrado, apresentando desde fisionomias florestais (Cerradão) até as campestres (campo limpo), distribuídas em mosaicos ao longo da paisagem. Ao longo do rio Araguaia existem pequenos remanescentes de floresta tropical úmida, e no extremo norte e sudeste, floresta estacional semidecidual. Neste Estado, além do cerrado, ocorrem três importantes áreas de transição ou ecótonos. Estas áreas de transição são: floresta amazônica - cerrado, cerrado - caatinga e cerrado - savana úmida.

A principal atividade agropecuária no Estado é a pecuária extensiva tradicional, principalmente em pastos naturais e/ou plantados, associados à agricultura de subsistência, ao extrativismo.

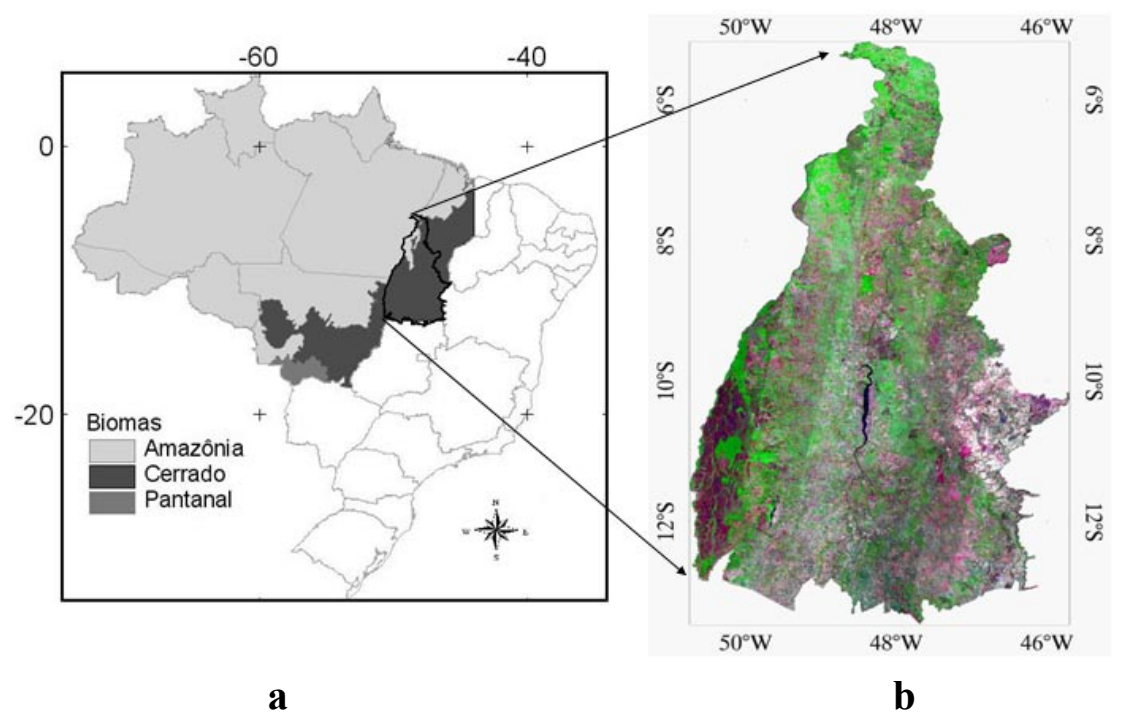

Figura 1 - Identificação dos Biomas da Amazônia Legal Brasileira (a). A área em cinza escuro corresponde ao Bioma Cerrado e o polígono delimitado na cor preta destaca a área de estudo (Estado de Tocantins), que pode se observar com maior detalhe na composição colorida (b) gerada a partir de dados do MODIS, canais 1, 2 e 7, período 09/06/2006 a 24/06/2006 (composição de 16 dias). 


\subsection{Materiais}

Os principais dados utilizados para analisar o padrão sazonal das classes de UCT correspondem a dois índices de vegetação (IV) do MODIS, isto é, o Índice de Vegetação de Diferença Normalizada (NDVI) e o Índice Melhorado de Vegetação (EVI). Para cada índice foram obtidas 12 imagens que representam os 12 meses do ano (2004). Os códigos das datas destas imagens são: 2004001, 2004033, 2004081, 2004097, 2004129, 2004161, 2004193, 2004241, 2004273, 2004305, 2004337 e 2004353. Cada imagem representa um período de 16 dias. Para cobrir a área de estudo, duas cenas (tiles) de cada período foram agrupadas (h13v09 e h13v10) formando um mosaico.

Dois conjuntos de dados foram usados para determinar a sazonalidade da precipitação na área de estudo. O primeiro incluiu dados diários do ano de 2004 obtidos a partir das estações meteorológicas, combinados com dados do satélite TRMM (Tropical Rainforest Measuring Mission) 3B42, com resolução espacial de $0,25^{\circ}$. Esta fusão teve o intuito de completar as áreas que não dispunham de estações de campo com os dados disponíveis de satélite, resultando numa imagem de precipitação diária do ano de 2004, interpolada para uma resolução de $0,02^{\circ}$.

O segundo conjunto de dados é uma série histórica longa de precipitação diária para o Brasil disponibilizada pelo CPC (Climate Prediction Center) da NCEP/NCAR, a qual foi elaborada considerando bases de dados providas pelo $\mathrm{CMCD} /$ INPE, INMET, FUNCEME/CE, LMRS/PB, EMPARN/RN, DMRH/PE, DHME/PI, CEPES/SE, NMRH/AL, SRH/BA,
CEMIG-SIMGE/MG, SEAG/ES, SIMEPAR/PR e CLIMERH/

SC. Esta série cobre o período compreendido entre 01/01/1948 e 31/07/2005 e tem resolução espacial de $1^{\circ}$.

\subsection{Metodologia}

A metodologia utilizada consistiu na análise do padrão de sazonalidade da precipitação com a dinâmica sazonal das principais classes de uso e cobertura da terra (UCT), para o ano de 2004, no Estado de Tocantins.

As principais classes de UCT na área de estudo são: floresta, cerradão, cerrado stricto sensu, campo cerrado, agricultura e agricultura-pecuária. As quatro primeiras classes correspondem às principais formações vegetais, enquanto as duas últimas às principais classes de uso da terra na região. Foram selecionadas três pequenas áreas, determinadas por polígonos (vetores), para cada classe de UCT, conforme detalhado em Becerra e Alvalá (2006). A localização destas áreas por classe de UCT pode ser observada na Figura 2a.

A localização dos polígonos foi definida de forma aleatória e o tamanho de cada um dos polígonos foi definido usando o critério de maior área homogênea, isto é, aquela área homogênea (de uma mesma classe) em volta de um ponto, o qual foi determinado de forma aleatória. $\mathrm{O}$ tamanho de cada polígono por classe de uso e cobertura da terra, além da média e o desvio padrão, pode ser observado na Tabela 1 .

A determinação do padrão sazonal da vegetação e da precipitação para o ano de 2004 são apresentados nos seguintes subitens.

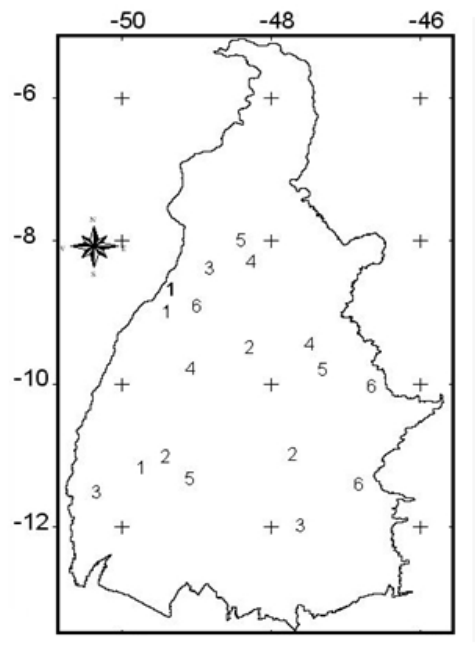

a

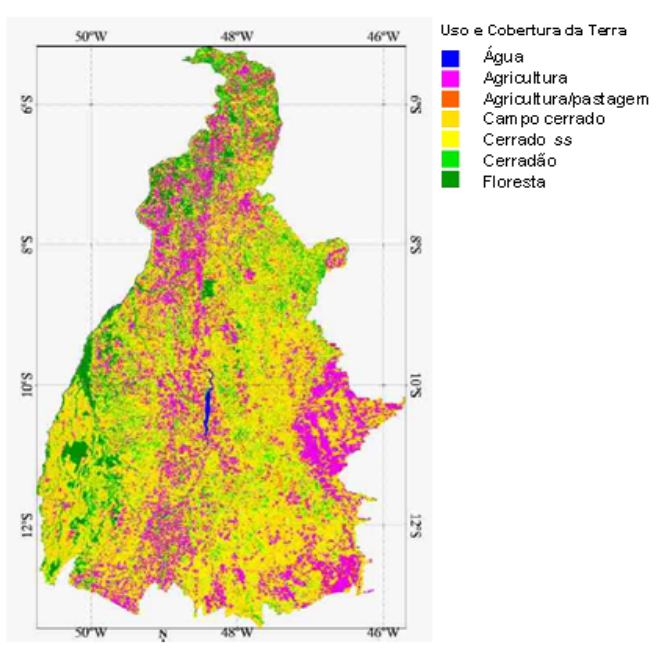

b

Figura 2 - Mapa da área de estudo com a localização dos polígonos, representados por números que indicam as classes de UCT avaliadas (a), e mapa do uso e cobertura da terra (b) segundo Becerra e Alvalá (2006). Em A os números 1, 2, 3, 4, 5 e 6 representam as classes floresta, cerradão, cerrado ss, campo cerrado, agricultura e agricultura-pastagem, respectivamente. 
Tabela 1 - Tamanho dos três polígonos, valor médio e desvio padrão para cada classe de uso e cobertura da terra, distribuídos ao longo da área de estudo, estado de Tocantins. A unidade dos números na tabela é o píxel, cada píxel corresponde a uma área de $250 \mathrm{~m}^{2}$.

\begin{tabular}{|l|c|c|c|c|c|}
\hline & Polígono 1 & Polígono 2 & Polígono 3 & Média & Desvio Padrão \\
\hline Agricultura-pastagem & 34 & 21 & 17 & 24 & 8,89 \\
\hline Agricultura & 32 & 39 & 22 & 31 & 8,54 \\
\hline Campo cerrado & 43 & 35 & 16 & 31,33 & 13,87 \\
\hline Cerrado ss & 12 & 17 & 16 & 15 & 2,65 \\
\hline Cerradão & 16 & 10 & 15 & 13,67 & 3,21 \\
\hline Floresta & 24 & 40 & 26 & 30 & 8,72 \\
\hline
\end{tabular}

\subsubsection{Padrão sazonal da vegetação}

Para avaliar a dinâmica sazonal da vegetação ao longo do ciclo anual de 2004 foram utilizados dois índices espectrais de vegetação do MODIS, o NDVI e o EVI.

O NDVI é uma relação entre medidas espectrais (refletância - $\rho$ ) de duas bandas, a do infravermelho próximo-IV $(700-1300 \mathrm{~nm})$ e a do vermelho-V $(600-700 \mathrm{~nm})$. Este índice é sensível à clorofila (Rouse et al., 1974). Os valores obtidos com o NDVI são contidos em uma mesma escala de valores, entre -1 e 1, utilizando-se a equação:

$$
\text { NDVI }=(\rho I V P-\rho V) /(\rho I V P+\rho V)
$$

O EVI foi desenvolvido para otimizar o sinal da vegetação, corrigindo distorções da luz refletida, causadas por material particulado suspenso no ar, assim como pela cobertura da terra embaixo do dossel da vegetação. Em áreas com grande quantidade de clorofila (abundante fitomassa foliar, como as florestas tropicais) o EVI não chega a se saturar facilmente como acontece com o NDVI. O calculado do EVI é feito através da seguinte equação:

$$
\begin{aligned}
& \mathrm{EVI}=\mathrm{G}(\mathrm{NIR}-\text { Vermelho }) / \\
& (\mathrm{L}+\mathrm{NIR}+\mathrm{C} 1 \text { vermelho }-\mathrm{C} 2 \text { azul })
\end{aligned}
$$

em que L é o fator de ajuste para os componentes abaixo do dossel (canopy background); C1 e C2 são os coeficientes usados para corrigir o espalhamento de aerossóis na atmosfera detectados pela banda vermelha usando a banda azul; e G é o fator de ganho. Os coeficientes adotados pelo algoritmo EVI (Huete et al., 1994; Huete et al., 1997) são: $\mathrm{L}=1, \mathrm{C} 1=6, \mathrm{C} 2$ $=7,5$ e $\mathrm{G}=2,5$.

O EVI é mais sensível às variações na resposta estrutural do dossel, incluindo o índice de área foliar (IAF), a fisionomia da planta e a arquitetura do dossel (Huete et al., 2002).

Estes índices (NDVI e EVI) oferecem dados temporais e espaciais consistentes sobre as condições da vegetação, de modo a monitorar a atividade fotossintética em suporte à detecção de mudança e interpretações biofísicas e estudo da fenologia.

O processamento dos dados índices de vegetação incluiu a elaboração de um mosaico e a re-projeção de duas cenas (h13v09 e h13v10) para cada data analisada. Em seguida, as imagens foram transformadas de $16 \mathrm{em} 8$ bits. Um polígono contendo os limites do Estado de Tocantins foi usado para extrair a área de estudo. Este processo foi feito com as imagens de cada uma das 12 datas analisadas, para cada índice. Finalmente, as 12 imagens de cada índice foram agrupadas (empilhadas) em um único arquivo. Neste arquivo foram sobrepostos os 18 polígonos (vetores), que correspondem às 6 classes de UCT, com o intuito de extrair os valores dos índices contidos em cada uma das 12 datas de cada índice. Estes valores, assim obtidos, foram plotados em um gráfico, que mostra no eixo $\mathrm{x}$ os meses do ano e no eixo y os valores (resposta) do índice. O resultado foi um gráfico para cada índice, que mostra o padrão sazonal da vegetação independentemente para o NDVI e para o EVI.

\subsubsection{Padrão sazonal da precipitação}

Os dados de precipitação diária de 2004 foram agrupados para o cálculo da precipitação mensal, que corresponde ao acumulado diário por mês, cujos cálculos foram feitos usando dados binários em ambiente do programa computacional GrADS. Os dados mensais foram exportados de binário para ASCII usando um programa em Fortran. Em seguida, estes dados ASCII, correspondentes às imagens de precipitação mensais, foram importados e convertidos em dados GEOTIFF, por um programa de análise de dados hiperespectrais (por exemplo, com o software SPRING 3.11).

Para determinar a precipitação mensal (2004) em cada uma das classes de UCT selecionada, gerou-se um arquivo (imagem) com 12 camadas de precipitação mensal. Da imagem de precipitação foram extraídos os valores de cada mês, usando os polígonos dos 18 vetores que correspondem às 6 classes de UCT analisados. 
Para determinar o padrão sazonal da precipitação para cada polígono analisado, considerou-se o processamento dos dados da série histórica de 1969 a 2005, seguindo-se as mesmas etapas adotadas com os dados de precipitação de 2004. Ou seja, a partir dos dados diários de precipitação da série histórica foram calculadas as médias mensais usando o programa GrADS. Os dados em formato binário foram transformados em ASCII usando um programa em Fortran. Os passos seguintes foram similares àqueles adotados com os dados de precipitação de 2004. O resultado da análise dos dados da série histórica (1969-2005) para cada classe de UCT foi um perfil sazonal de precipitação.

Para avaliar a relação da variação mensal da precipitação com a variação mensal dos índices de vegetação de cada um das classes de uso e cobertura avaliados, foram plotados num diagrama de dispersão os dados de precipitação no eixo das abscissas e os dados de vegetação do índice EVI (de cada uso e cobertura da terra) no eixo das ordenadas. Para cada classe de UCT foi ajustada uma linha de tendência usando uma equação de regressão linear, sendo a precipitação a variável independente e índice de vegetação a variável dependente. Desta maneira foi determinado para cada classe de uso e cobertura da terra o coeficiente de regressão $\left(R^{2}\right)$. Os valores deste coeficiente assim obtidos indicam o grau de relação entre a precipitação e o índice de vegetação EVI para cada um das classes de uso e cobertura da terra. Finalmente, ressalta-se que a análise foi feita considerando os dados de 2004; portanto, os resultados e interpretações correspondem somente para esse ano (2004).

\section{RESULTADOS E DISCUSSÕES}

\subsection{Padrão sazonal da precipitação}

Os perfis sazonais da precipitação obtidos a partir de dados da série histórica (período de 1969 a 2005) e anual (2004) para as seis classes de uso e cobertura da terra analisadas são apresentados na Figura 3.

Os resultados, ilustrados na Figura 3 a mostram que a estação seca, com precipitação inferior a $100 \mathrm{~mm}$, começa em maio e vai até o mês de setembro nas áreas que cobrem as 6 classes de UCT. Nesta estação, o padrão sazonal da precipitação nas áreas ocupadas pelas 6 classes de UCT são similares. Padrão similar, também foi observado na estação chuvosa nas áreas correspondentes à maioria dos tipos de UCT, apresentando apenas uma pequena diferença nas áreas de campo cerrado, onde as precipitações mínimas registradas foram inferiores às demais áreas no mês de dezembro.

Na Figura 3b, que ilustra o perfil da precipitação no ano de 2004, observa-se um padrão similar com o perfil da série histórica (Figura 3a) na estação seca. No entanto, no mês de julho de 2004 houve ocorrência de chuvas na maioria das classes estudadas, exceto nas áreas de cerradão. $\mathrm{Na}$ estação chuvosa houve uma diferenciação entre as áreas das 6 classes de UCT, com maior (menor) precipitação nas áreas de floresta (cerrado). Estes resultados mostram que a precipitação no ano de 2004 nas áreas de estudo, foi homogênea no período de secas, e heterogênea na época de chuvas, principalmente nos meses de outubro a dezembro.

Quanto à comparação dos dois perfis de precipitação correspondentes a série histórica (Figura 3a) e aquela do ano de 2004 (Figura 3b), a similaridade destes dois perfis é média, confirmada pela correlação de Pearson com $r=0,56$. Este valor sugere semelhanças no padrão geral sazonal da precipitação para a região, e diferenças específicas nos períodos de seca e chuva, entre os dados de 2004 (Figura 3b) e os dados do padrão climático de precipitação da região (Figura 3a). Entre as características específicas de 2004 temos: na estação seca um período mais prolongado, mas com precipitações no meio deste período, e na estação de chuvas a precipitação é maior do que a média em janeiro, novembro e dezembro.

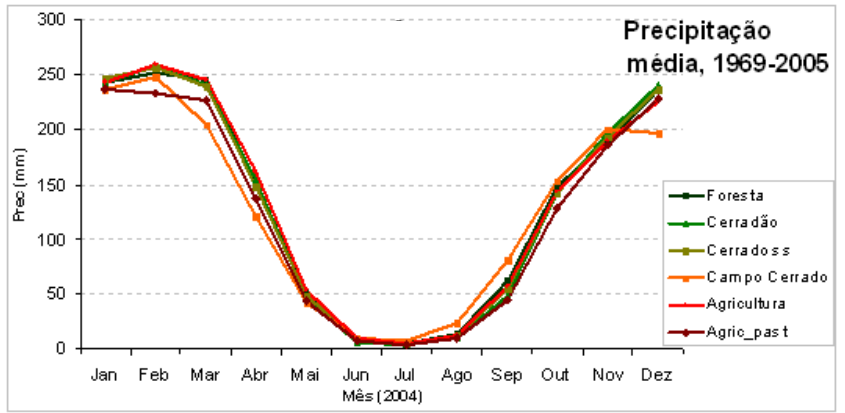

a

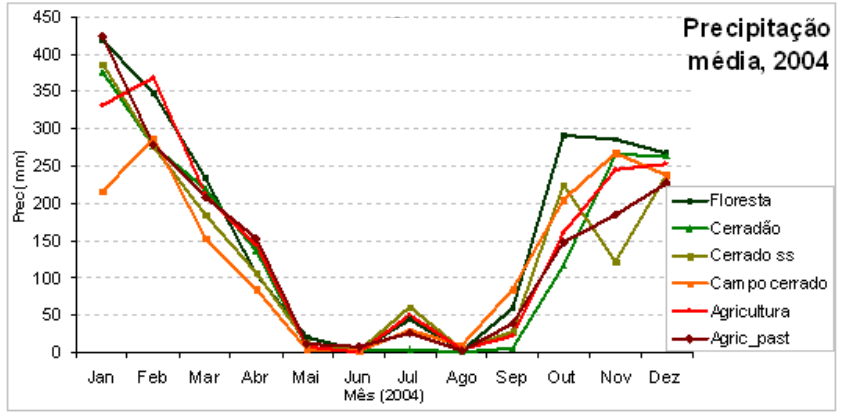

b

Figura 3 - Padrão sazonal de precipitação determinado a partir da análise de uma série longa (1969 a 2005) e de dados de 2004, representados nas figuras a e b respectivamente. As classes avaliadas são: floresta; cerradão; cerrado stricto sensu; campo cerrado; agricultura; agricultura-pastagem. 
Quanto à diferenciação específica dos perfis de precipitação entre as classes analisadas, pode-se notar que na análise climatológica (período 1969 a 2005) os perfis das classes apresentam-se muito próximos e/ou sobrepostos. $\mathrm{Na}$ análise anual (2004) os perfis encontram-se separados, principalmente no período de chuvas. Estas diferenças de padrões nos perfis se devem principalmente ao tipo de dado utilizado, sendo na análise climatológica de menor resolução espacial, que tende a uniformizar as respostas da precipitação, e na análise anual de maior resolução espacial, que tende a diferenciar melhor a resposta da precipitação.

\subsection{Padrão sazonal da vegetação}

O perfil sazonal da dinâmica da vegetação nas áreas que correspondem às 6 classes de UCT, a partir das análises do NDVI e do EVI para o ano de 2004 são apresentados na Figura 4. O comportamento sazonal das classes de UCT usando estes índices mostrou alta atividade fotossintética e fitomassa foliar na estação chuvosa (janeiro a março e outubro a dezembro de 2004), com diminuição gradual na época seca.

Os resultados da Figura 4A mostram o perfil das classes de UCT usando o índice EVI. Nas áreas que representam as classes de UCT (cerradão, cerrado ss, campo cerrado, agricultura e agricultura-pastagem) observa-se um decréscimo dos valores do índice, que vão da estação de chuvas (inicio do ano) à estação seca, com os menores valores do índice no mês de agosto. No caso das áreas de florestas, o perfil sazonal ao longo do ano de 2004 não mostrou decréscimos observados nas outras classes de UCT.

Na Figura 4b, que mostra o perfil sazonal da vegetação usando o índice NDVI para o ano de 2004, observa-se um padrão similar com os resultados obtidos com o NDVI (Figura 4a) em termos de tendência geral. No entanto, os menores valores deste índice, que correspondem à estação seca, não coincidiram nas 6 classes de UCT, sendo que em 3 classes (cerradão, cerrado ss e campo cerrado) o menor valor foi obtido para o mês de agosto enquanto em 2 classes (agricultura e agricultura-pastagem) o menor valor ocorreu em setembro. Nesta mesma figura, pode-se notar que a maioria dos valores das classes avaliadas no mês de fevereiro, apresentou uma queda acentuada, quando comparada com os valores do mês anterior e do mês posterior. Isto se deve principalmente ao efeito da maior cobertura de nuvens deste mês, que gera esse tipo de resultado anômalo com conseqüente diminuição dos valores do NDVI das classes de UCT, as quais, nessa época do ano (estação de chuvas), apresentam naturalmente valores altos.

$\mathrm{Na}$ comparação dos resultados dos dois índices de vegetação, pode-se observar que o EVI mostra maior relação com o padrão de precipitação, se comparado com o NDVI. Assim, no mês de agosto foi registrada a menor precipitação e também os menores valores do EVI, que indicam menor biomassa no dossel das classes de UCT analisados. No caso do NDVI, os menores valores foram registrados nos meses de agosto e setembro, o que indica que a atividade fotossintética das classes de UCT relacionada com este índice, sofre o impacto do estresse hídrico nesses dois meses, devido provavelmente às limitações hídricas, em conformidade com o mostrado por Huete et al. (2006).

Nas áreas que representam a classe floresta, a análise do índice EVI indica que não houve decréscimos significativos na estação seca. A análise da classe floresta versus o padrão do índice NDVI, mostra uma pequena queda da fitomassa foliar no mês de setembro com relação aos outros meses, bem como uma pequena queda desta classe com relação às outras classes, cuja perda de fitomassa foliar é mais acentuada, sendo esta uma das principais características da vegetação savânica. No caso da classe floresta, a pequena queda da fitomassa foliar pode ser explicada pela disponibilidade hídrica atingida pelas raízes profundas destas árvores e/ou pela proximidade de cursos de

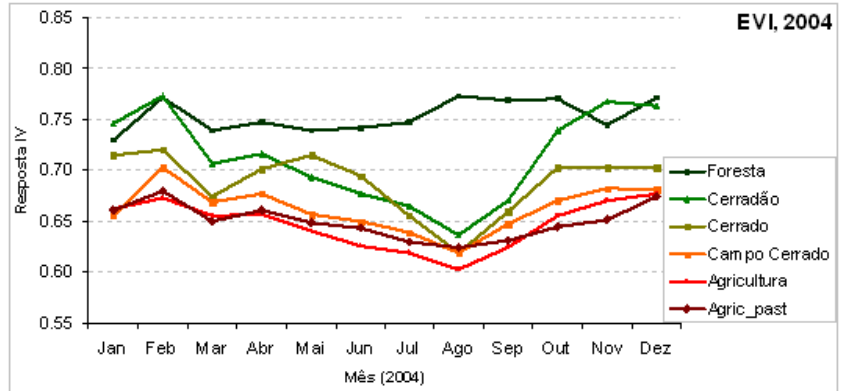

a

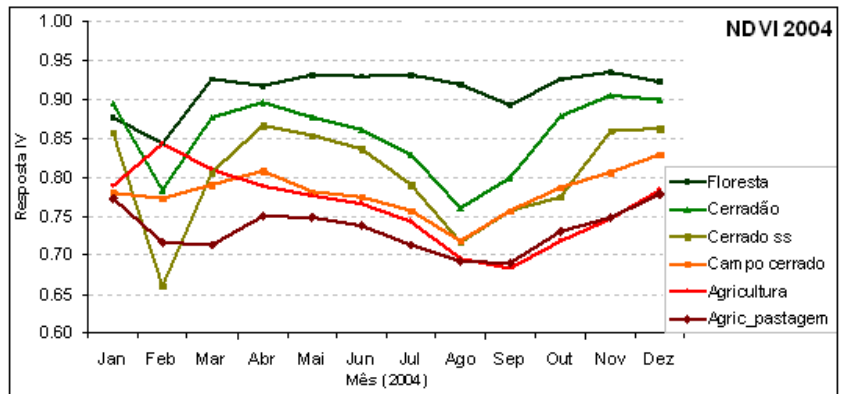

b

Figura 4 - Padrão sazonal da vegetação no ano de 2004 a partir da análise de dois índices de vegetação, NDVI e EVI, relacionados com atividade fotossintética e fitomassa no dossel. As classes avaliadas são: floresta; cerradão; cerrado strictu sensu; campo cerrado; agricultura; agriculturapastagem. 
rios (mata ciliar). Segundo Huete et al. (2006), a disponibilidade hídrica das áreas de floresta propiciaria uma estabilidade na atividade fotossintética e conseqüente produção de fitomassa foliar mantida (ou com pequena queda) na época seca.

\subsection{Relação da precipitação com as classes de uso e cobertura da terra}

Os resultados da quantificação da relação das classes de uso e cobertura da terra com a precipitação são apresentados na Figura 5. Nesta Figura, as linhas coloridas representam o ajuste da linha de tendência entre a precipitação e cada classe de uso e cobertura da terra obtida a partir de uma equação de regressão linear simples. Os coeficientes de regressão (R2) das classes floresta, cerradão cerrado ss, campo cerrado, agricultura e agricultura-pastagem foram 0,0007 ; $0.7357 ; 0,3093 ; 0,6151 ; 0,5475$ e 0,7518 , respectivamente.

Os valores dos coeficientes de regressão acima mencionados confirmam que as classes de uso e cobertura da terra na região do cerrado apresentam um padrão sazonal relacionado com a sazonalidade da precipitação e que a floresta é a única classe que tem um comportamento diferenciado, com a precipitação não tendo uma relação direta com a cobertura vegetal.

Estes resultados indicam uma relação positiva entre a variável precipitação e os índices de vegetação NDVI e EVI das principais classes de vegetação que caracterizam o Cerrado (cerradão, cerrado ss e campo cerrado) e das classes de uso da terra (agricultura e agricultura-pastagem) ao longo de um ciclo anual (2004), sinalizando a importância de inclusão de variáveis ambientais, no caso a precipitação, que permita auxiliar na identificação das principais classes de cobertura vegetal na região do Bioma Cerrado da Amazônia Legal.
Finalmente, as classes de UCT avaliadas, quando incorporadas aos mapas de vegetação utilizados pelos esquemas de superfície (p.e, o modelo simplificado da biosfera - SSiB) e acoplados a modelos meteorológicos, poderão melhorar os prognósticos e as previsões de tempo e clima da região estudada.

\section{CONCLUSÕES}

A inclusão de dados de precipitação, na interpretação do comportamento sazonal da vegetação na região do Bioma Cerrado, permitiu compreender de forma mais exata a dinâmica sazonal das diferentes classes de uso e cobertura da terra no ano de 2004. A dinâmica sazonal da vegetação usando índices de vegetação, NDVI e EVI, permitiu identificar o padrão sazonal das principais classes de uso e cobertura da terra no ano de 2004. O índice EVI mostrou maior relação com a precipitação na detecção do período com menor produção de fitomassa foliar nas classes de UCT. Na análise sazonal da vegetação, o NDVI mostrou melhor separabilidade e identificação das classes de UCT ao longo do ciclo anual, excetuando o início do ano, mês de fevereiro, quando houve uma contaminação da resposta espectral dos alvos por conta da maior nebulosidade desse período. As áreas de floresta mostraram um padrão diferente das demais classes de UCT analisadas. Na época chuvosa a atividade fotossintética e produção de fitomassa foliar, foram semelhantes à época seca, com uma leve queda no mês de setembro devido presumivelmente ao efeito retardado do período seco. A análise do padrão sazonal das classes de UCT no Bioma Cerrado permitiu identificar os melhores meses para diferenciar fisionomias vegetais com respostas espectrais similares, difíceis de identificação em mapeamentos sem dimensão sazonal. A freqüência regular de dados IV (índices de

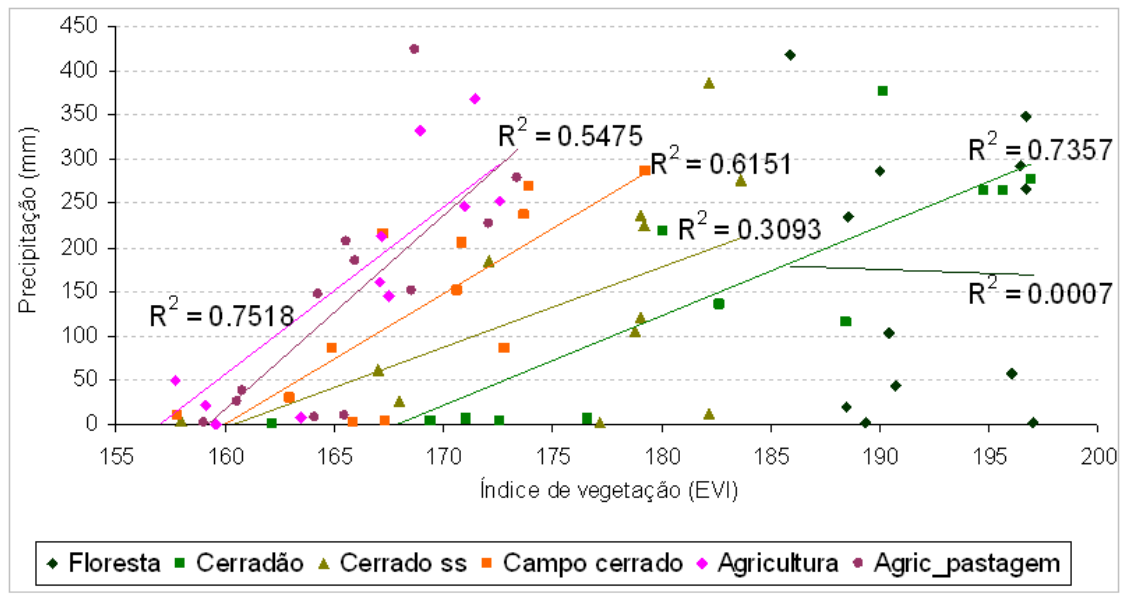

Figura 5 - Relação da precipitação com cada classe de uso e cobertura da terra na região do Cerrado no Estado de Tocantins. 
vegetação) do MODIS permitiu elaborar séries temporais que facilitam o acompanhamento do ciclo sazonal da vegetação. $O$ EVI permitiu analisar melhores períodos com maior cobertura de nuvens, enquanto o NDVI permitiu separar melhor as classes de UCT ao longo do ciclo anual.

\section{AGRADECIMENTOS}

Este trabalho foi desenvolvido no escopo da Rede GEOMA, na componente Modelagem Climática para a Amazônia. O autor Jorge Alberto Bustamante Becerra agradece a Rede GEOMA pela bolsa PCI, modalidade DTI. Os autores agradecem aos pesquisadores Paulo Yoshio Kubota e Jose Roberto Rozante pelo apoio na elaboração dos dados de precipitação.

\section{REFERÊNCIAS BIBLIOGRÁFICAS}

BECERRA, J.B.; ALVALÁ, R.C.S. Detection of Tropical Savannah (Cerrado) Physiognomies in the Legal Amazon by the Application of the Vegetation and Moisture Indices with MODIS Time Series Data. In: The 8th International Conference On Southern Hemisphere Meteorology And Oceanography, Anais, Foz de Iguaçu. Proceedings of ICSHMO, Brazil, v. 1, p. 861-868, 2006.

BOX, O. Macroclimate and plant forms : an introduction to predictive modelling in phytogeography, Junk, The Hague. 1981.

CHAMPEAUX, J.L; ARCOS, D; BAZILE, E; GIARD, D; GOUTORBE, J; HABETS, F; NOILHAN, J; ROUJEAN, J. AVHRR-derived vegetation mapping over Western Europe for use in numerical weather prediction models. Int. J. Remote Sens, v. 21, 1183-1199, 2000.

CHARNEY, J.G.; QUIRK, W.J.; CHOW, S.H.; KORNFIELD, $\mathrm{J}$. A comparative study of the effects of albedo change on drought in semi arid regions. J. Atmos. Sci, v. 34, 1366$1385,1977$.

COUTINHO, L.M. Fire in the ecology of the Brazilian Cerrado. In: GOLDHAMMER, J.G. (ed). Fire in the Tropical Biota, Springer-Verlag, 82-105, 1990.

DeFRIES, R.S.; TOWNSHEND, J.R.G.; HANSEN, M.C. Continuous fields of vegetation characteristics at the global scale at $1 \mathrm{~km}$ resolution. J. Geophys. Res, v. 104(D14), 16911-16923, 1999.

FERREIRA, LG.; YOSHIOKA H.; HUETE, A.; SANO, E. Seasonal landscape and spectral vegetation index dynamics in the Brazilian Cerrado: An analysis within the Large-Scale Biosphere-Atmosphere Experiment in Amazônia (LBA), Remote Sens. Environ, v. 87, 534-550, 2003.

FERREIRA, L.G.; YOSHIOKA, H.; HUETE, A.; SANO,
E.E. Optical characterization of the Brazilian Savanna physiognomies for improved land cover monitoring of the cerrado biome: preliminary assessments from an airborne campaign over an LBA core site. J. Arid. Environ, v. 56, 425-447, 2004.

HALL, F.G.; TOWNSHEND, J.R.; ENGMAN, E.T. Status of remote sensing algorithms for estimation of land surface state parameters. Remote Sens. Environ, v. 51, 138-156, 1995.

HOFFMANN, W.A.; JACKSON, R.B. Vegetation-climate feedbacks in the conversion of tropical savanna to grassland. J. Climate, v. 13, 1593-1602, 2000.

HUETE, A.; JUSTICE, C.; LIU, H. Development of vegetation and soil indices for MODIS-EOS. Remote Sens. Environ, v. 49, 224-234, 1994.

HUETE, A.R.; LIU, H.Q.; BATCHILY, K.; VAN LEEUWEN, W. A comparison of vegetation indices over a global set of TM images for EOS-MODIS. Remote Sens. Environ, v. 59(3), 440-451, 1997.

HUETE, A.R.; DIDAN, K.; MIURA, T.; RODRIGUEZ, E.P.; GAO, X.; FERREIRA, L.G. Overview of the radiometric and biophysical performance of the MODIS vegetation indices. Remote Sens. Environ, v. 83, 195-213, 2002

HUETE, A.R.; DIDAN, K.; SHIMABUKURO, Y.; RATANA, P.; SALESKA, S.R.; HUTYRA, L.R.; YANG, W.; NEMANI, R.R.; MYNENI, R. Amazon rainforests greenup with sunlight in dry season. Geophys. Res. Lett, v. 33, L06405, 2006.

INSTITUTO BRASILEIRO DE GEOGRAFIA ESTATÍSTICA (IBGE). Manual técnico de vegetação brasileira, Rio de Janeiro, 1992. $92 \mathrm{p}$.

NIMER, E. Clima. In: IBGE. Geografia do Brasil/Região Sudeste v.3. Rio de Janeiro: FIBGE, 1977.

RATANA, P.; HUETE, A. Seasonal dynamics of native and converted cerrado physiognomies with MODIS data. Geoscience and Remote Sensing Symposium, 2004. IGARSS '04. Proceedings, Anais, 2004 IEEE International, 4336-4339, 2004.

RATTER, J.A.; RIBEIRO, J.F.; BRIDGEWATER, S. The Brazilian cerrado vegetation and threats to its biodiversity. Annals of Botany, v. 80, 223-230, 1997.

ROUSE, J.W.; HAAS, R.H.; SCHELL, J.A.; DEERING, D.W. Monitoring vegetation systems in the Great Plains with ERTS. NASA. Goddard Space Flight Center 3d ERTS-1 Symposium, v. 1, Sect. A, p 309-317 Proceedings, Third Earth Resources Technology Satellite-1 Symposium, Anais, Greenbelt: NASA SP-351 I, 309-317, 1974.

SELLERS, P. J; MINTZ, Y.; SUD, Y. C.; DALCHER, A. A Simple Biosphere Model (SiB) for use within general circulation models. J. Atmos. Sci, v. 43, 505-531, 1986. 
SELLERS, P.J.; HEISER, M.D.; HALL, F.G.; VERMA, S.B.; DESJARDINS, R.L.; SCHUEPP, P.M.; MACPHERSON, J.I. The impact of using area-averaged land surface properties -topography, vegetation condition, soil wetness In calculations of intermediate scale (approximately $10 \mathrm{~km} 2$ ) surface-atmosphere heat and moisture fluxes. J. Hydrol, v. 190, 3-4, pp. 269-301, 1997.

SESTINI, M.F.;ALVALÁ, R.C.S.; MELLO, E.K.;VALERIANO, D.M.; CHAN, C.S.; NOBRE, C.A.; PAIVA, J.A.C.; REIMER, E.S. Elaboração de Mapas de Vegetação para Utilização em Modelos Meteorológicos e Hidrológicos. São José Campos. INPE, 2002. 74 p. (INPE-8972-RPQ/730).

SHUKLA, J.; MINTZ, Y. Influence of land surface evapotranspiration on the earth's climate. Science, v. 251, 1498-1501, 1982.

SHUKLA, J.; NOBRE, C.; SELLERS, P. Amazon deforestation and climate change, Science, v. 247, 1322-1325, 1990.
SUD, Y.C.; SHUKLA, J.; MINTZ, Y. Influence of land surface roughness on atmospheric circulation and rainfall: A sensitivity study with a general circulation model. J. Appl. Meteorol, v. 27, 1036-1054, 1988.

SUD, Y.C.; YANG, R.; WALKER, G.K. Impact of in situ deforestation in Amazon on the regional climate: general circulation model simulation study. J. Geophys. Res, v. 101 (D3), 7095-7109, 1996.

WALTER, H. Vegetation of earth, in relation of climate and the ecophysiological conditions, English University Press, London. 1973.

WILSON, M.F.; HENDERSON-SELLERS, A global archive of land cover and soils data for use in general circulation climate models. J. Climatol, v. 5, 119-143, 1985.

XUE, Y.; SHUKLA, J. The influence of land surface properties on Sahel climate. I, desertification. J. Climatol, v. 6, 22322245, 1993. 